Chirurg 2017· 88:93-94

DOI 10.1007/s00104-016-0350-7

C) Springer Medizin Verlag Berlin 2016

CrossMark

\title{
T. Pohlemann
}

Klinik für Unfall-, Hand- und Wiederherstellungschirurgie, Universitätsklinikum des Saarlandes, Homburg/Saar, Deutschland

\section{Chirurgie im Alter}

Die Durchführung operativer Eingriffe war in der Pionierzeit der modernen Chirurgie allenfalls jungen und gesunden $\mathrm{Pa}$ tienten vorgehalten. Noch vor 50 Jahren wurde heftig über die ethischen Grenzen bei der Durchführung von Operationen bei geriatrischen Patienten diskutiert. Der schnelle technische Fortschritt, die Spezialisierung innerhalb der chirurgischen Fächer, aber letztendlich auch der zunehmende Bedarf im Rahmen des demographischen Wandels haben diese Grenzen erheblich verschoben und neue Bedürfnisse geschaffen. Der hohe Aktivitätsgrad vieler geriatrischer Patienten sowie eine gute körperliche und geistige Konstitution bedingen einen hohen Anspruch dieser Patienten an das therapeutische Vorgehen.

Während noch 1992 in E. Letournels Standardwerk über die operative Versorgung von Azetabulumverletzungen ein Alter über 60 Jahre und/oder das Vorliegen einer Osteoporose als Kontraindikationen für die operative Rekonstruktion dieser Verletzungen angegeben wurden, zeigen die Daten des Beckenregisters der Deutschen Gesellschaft für Unfallchirurgie, dass zwischenzeitlich über die Hälfte der Patienten in diese Kategorie fallen und der Aktivitätsgrad dieser Patienten in der Mehrzahl eine operative Versorgung rechtfertigt. Allerdings werden dazu neue operative Konzepte benötigt.

Wir haben daher für die Kongressausgabe von Der Chirurg zum DGCHJahreskongress 2017 dieses Leitthema gewählt und berufene Autoren aus unterschiedlichen chirurgischen Fachgebieten gebeten, das Thema "Alterschirurgie“ aus ihrem fachspezifischen Blickwinkel darzustellen. Es sollte dabei nicht nur auf operationstechnische Details fokussiert werden, sondern auch untersucht werden, wie strukturelle Änderungen bei der
Versorgung geriatrischer Patienten aussehen könnten. Eine Vollständigkeitkann bei der Breite des Themas naturgemäß nicht beansprucht werden. Wir hoffen aber, dass die Auswahl der Themen und die kompetente Darstellung die verschiedenen Facetten der "Chirurgie im Alter“ erkennen lassen. Sicherlich bedarf es einem interdisziplinären Ansatz, um neue Konzepte und Techniken zu entwickeln.

Die Untersuchungen der Arbeitsgruppe Raschke et al. aus Münster beschäftigen sich mit dem „Delir“ als ein in der Alterstraumatologie häufiges und bisher noch nicht ausreichend gelöstes Problem. Der Behandlung des Delirs muss interdisziplinär und auch interprofessionell begegnet werden. Neben dem rein medizinischen Problem hat die Prävention sowie die schnelle, spezifische Frühbehandlung auch erhebliche soziale und ökonomische Konsequenzen im Hinblick auf die idealerweise angestrebte frühe Rehabilitation und Reintegration der geriatrischen Patienten. Das in Münster erprobte Konzept ist dabei ein gangbarer Lösungsansatz. Besonders interessant erscheint auch die Analyse der notwendigen strukturellen Vorhaltungen und der ggf. auch sektorenübergreifenden Vernetzung für eine effektive, hochqualitative und erfolgreiche Betreuung verunfallter geriatrischer Patienten.

Ein spezielles und zunehmendes Problem stellen die periprothetischen Azetabulumfrakturen dar. Der zunehmende Anteil geriatrischer Patienten mit vorbestehendem totalendoprothetischem oder auch Teilhüftersatz führt bei Stürzen zwangsläufig auch zu einer steigenden Zahl von Patienten mit Frakturen um die Hüftpfanne. In der eigenen Klinik wurden dazu spezielle Ablaufkonzepte etabliert und die Operationstechniken angepasst. Größere Serien können noch 
nicht präsentiert werden, aber die von Herath et al. präsentierten Konzepte helfen bei der Entscheidungsfindung und Durchführung therapeutischer Maßnahmen.

Wiegmann et al. geben einen Überblick über die Herzchirurgie im hohen Alter. Auch hier gibt es notwendige Indikationsanpassungen bei den zwischenzeitlich immer aktiveren „Alterspatienten“, um die Lebensqualität und Selbstständigkeit zu erhalten. Der schon länger bekannten Abkehr des „kalendarischen Alters“ zur Indikationsbegrenzung kommt gerade in der Herzchirurgie eine besondere Bedeutung zu. Auch der Aspekt der Operation in einer frühen, oftmals noch symptomarmen oder sogar symptomfreien Phase bekommt eine besondere Bedeutung. Auch hier haben sich interdisziplinäre Teams für die Indikationsstellung und Therapieplanung bewährt.

Kaschwich und Koautoren aus der Arbeitsgruppe E. S. Debus aus Hamburg beleuchten den rapiden Wandel in der Indikationsstellung und Verfahrenswahl bei der Behandlung vaskulärer Erkrankungen geriatrischer Patienten. Insbesondere der schnelle Fortschritt endovaskulärer Techniken verschiebt die Indikationsgrenzen erheblich und lässt zwischenzeitlich auch bei schweren Begleiterkrankungen, die früher als Kontraindikationen angesehen wurden, eine erfolgreiche gefäßchirurgische Therapie zu.

Glanemann und Schuld legen eine eigene Analyse von Behandlungen von 1334 Patienten mit kolorektalen Tumoren vor. Nach altersabhängiger Stratifizierung wurde neben Lokalisation, Indikation und Operationstechnik insbesondere die Rate der perioperativen Komplikationen untersucht. Die Autoren kommen zum Schluss, dass bei geriatrischen Patienten erwartungsgemäß erhöhte Morbiditäts- und Mortalitätsraten $\mathrm{zu}$ beobachten waren, wobei hierbei insbesondere Wundinfekte und kardiovaskuläre Komplikationen eine führende Rolle einnahmen. Die Rate der Anastomoseninsuffizienz zeigte keine Korrelation zum Alter. Als Fazit wird auch hier auf die Notwendigkeit der interdisziplinären Evaluation und Betreuung der Patienten hingewiesen, insbesondere auch unter Einbeziehung geriatrischer Kompetenz.

Juratli et al. beleuchten das chronische Subduralhämatom im Alter. Internationale Studien zeigen eine deutliche $\mathrm{Zu}$ nahme dieses Krankheitsbildes im Alter im Verlauf der letzten Jahre, was insbesondere auf die zunehmende Einnahme von gerinnungshemmenden Medikamenten zurückgeführt wird. Die Autoren diskutieren Symptomatik und Diagnostik und geben einen Überblick über die aktuellen Therapiekonzepte. Insbesondere der Methode der sog. „TwistDrill-Kraniostomie“ als minimalinvasive Maßnahme, die teilweise sogar in Lokalanästhesie durchgeführt wird, kommt bei geriatrischen Patienten eine besondere Bedeutung zu. Auch ganz neue Ansätze einer nichtoperativen, medikamentösen Therapie werden diskutiert, auch wenn die Studienlage hierzu noch sehr eingeschränkt ist.

Abschließend widmet sich H. Eichler der übergreifenden Problematik des Gerinnungsmanagements bei geriatrischen Patienten. Die hohe Inzidenz der Einnahme gerinnungshemmender Medikamente, insbesondere die neuen oral einzunehmenden Substanzklassen, in der betreffenden Bevölkerungsgruppe stellt vor allem in der Akutchirurgie ein Problem dar. Aber auch aktuelle Konzepte der perioperativen Thromboembolieprophylaxe werden eindrücklich dargestellt und hochkompetent kommentiert.

Ich danke allen Autoren für die Erstellung der exzellenten Beiträge. Sie werden sicherlich dazu beitragen, dass einerseits für die in der täglichen Praxis immer häufiger auftretenden Probleme der geriatrischen Patienten sensibilisiert wird, andererseits aber auch dazu ermutigen, neue Konzepte für die Erhaltung der Lebensqualität in dieser zahlenmäßig rasch zunehmenden Bevölkerungsgruppe zu etablieren. Dem interdisziplinären Ansatz wird dabei sicher eine besondere Bedeutung zukommen!

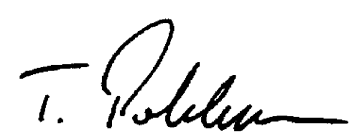

Prof. Tim Pohlemann

\section{Korrespondenzadresse}

\section{Prof. T. Pohlemann}

Klinik für Unfall-, Hand- und Wiederherstellungschirurgie, Universitätsklinikum des Saarlandes

Kirrberger Str. 1, 66421 Homburg/Saar,

Deutschland

tim.pohlemann@uks.eu

Interessenkonflikt. T. Pohlemann gibt an, dass kein Interessenkonflikt besteht. 